\title{
EL MÉTODO DE CULTIVO “IN VITRO” COMO INSTRUMENTO PRÁCTICO EN EL DIAGNÓSTICO Y EL AISLAMIENTO PRIMÁRIO DE LEISHMANIA BRAZILIENSIS BRAZILIENSIS I. OBSERVACIONES DE LABORATÓRIO
}

\author{
Eduardo M. Netto, Cesar C. Cuba, Philip D. Marsden y Air C. Barretto
}

\begin{abstract}
Comparamos diferentes medios artificiales y procedimientos de cultivo in vitro de Leishmania braziliensis braziliensis (Lbb) aislada de lesiones de hamsteres experimentalmente infectados. Observamos que la aspiración de material de la lesión, usando jeringa de $5 \mathrm{ml}$ y aguja $22 \mathrm{~g}(25 \times 8 \mathrm{~mm})$, mostró resultados satisfactorios siendo un procedimiento conveniente de colecta. El medio de Agar Sangre Difco (DAB) es un medio eficiente para el aislamiento de Lbb. El medio de Evans demostró similares resultados al DAB, mientras que el medio liquido de Schneider y el quimicamente definido $A R-103$ fueron nitidamente inferiores.

En la propagación in vitro de Lbb, el medio de Schneider permitió un buen crecimiento del flagelado, alcanzando densidades de $0,21-2,57 \times 10^{8}$ promastigotes por $\mathrm{ml}$ ya, en el 5. día de cultivo. La adición de 5-fluorocytosine a los tubos de cultivo $(150 \mu \mathrm{g} / \mathrm{ml})$ para evitar la contaminación por hongos, no inhibió el crecimiento de Lbb.
\end{abstract}

Palabras claves: Leishmania braziliensis braziliensis. Cultivo in vitro.

Aislar el agente causal de las leishmaniasis es necesario no solamente para el diagnóstico como para la identificación de la sub-espécie de Leishmania infectante ${ }^{19}$. En los estudos quimioterapéuticos de la enfermedad es también valioso intentar aislar el parásito posttratamiento para probar, en forma incuestionable, el éxito o fracaso de un determinado compuesto antileishmanial 16.

Es en relación a la correcta elección del método más apropriado de aislamiento del parásito que debemos adoptar una decisión práctica y eficiente. Este laboratório ha reportado, muy recientemente, sus hallazgos en la demonstración directa de Leishmania braziliensis braziliensis en pacientes con lesiones leishmaniásicas cutáneas y mucocutaneas, prevalentes en Très Braços, Bahia, Brasil ${ }^{9}$. En dichos estudios, la inoculación en hamsteres dorados Mesocricetus auratus fué el procedimiento más eficiente que el método de cultivo "in vitro" para el diagnóstico y aislamiento primário de ésta sub-espécie de Leishmania. Sin embargo, debemos anotar que, en esos estudios iniciales tuvimos serios problemas de contaminación bacteriana, por hongos y levaduras, que hicieron fracasar el método cultivo.

Núcleo de Medicina Tropical e Nutrição, Universidade de Brasília. 70910 - Brasilia, DF.

Pesquisa financiada parcialmente pelo RE-GRANT 820526 WHO/TDR e CNPq.

United States Public Service Grant (AI/16282-04), Department of International Medicine Cornell Medical College. New York.

Recebido para publicação em 18/9/1985
Posteriormente, alertados por las publicaciones de Miles ${ }^{17}$ y Palomino et alii ${ }^{18}$ pasamos a utilizar gentamicina y 5-fluorocytosine, reduciendo mucho los índices de contaminación de los cultivos y los resultados de éstos estudios son presentados en ésta serie de trabajos.

La presente investigación formuló los dos objetivos siguientes para ganar experiencia laboratorial que serviria de base a futuras investigaciones de campo:

1) Aislar $L . b$. braziliensis y $L$. mexicana de hamsteres experimentalmente infectados, por medio de cultivos. Comparar la sensibilidad de medios y métodos de aislamiento.

2) Lograr el crecimiento in vitro de los promastigotes de esa sub-especie de Leishmania, para estudios de texonomía bioquimica e inmunologica.

\section{MATERIAL Y MÉTODOS}

1. Medios de Cultivo empleados:

A) Medio TC 199 (cultivo de células de mamiferos) Gibco, Labs., N.Y. USA, suplementado con $20 \%$ de suero bovino fetal (Sterile System Hy-Clone-USA) inactivado, por 30 minutos, a $56^{\circ} \mathrm{C} .14$

B) Medio de Schneider (cultivo de células de insetos) Drosophila Gibco medium, Gibco. N.Y. USA. complementado con el mismo suero bovino fetal como en (A) 1213 .

C) Medio agar sangre Difco (DAB), preparado como recomendado por Walton et al. ${ }^{22}$, y $15 \%$ de sangre desfibrinada de conejo. La fase líquida constituida por suero fisiológico, $0.85 \%$. 
Netto EM, Cuba CC, Marsden PD, Barreto AC. El método de cultivo "in vitro" como instrumento práctico en el diagnóstico yel aislamiento primário de Leishmania braziliensis braziliensis. I. Observaciones de laboratório. Revista da Sociedade Brasileira de Medicina Tropical 19: 79-84, Abr-Jun, 1986

D) Mediode Evans (Evans modified Tobie's Medium). Medio preparado como fue indicado por Evans 1011.

E) Medio químicamente definido AR-103. Cedido por el Dr. H. Azevedo para los estudios en Leishmania y con la formulación publicada por Azevedo y Roitman ${ }^{1}$.

2. Aislamiento primario de L. b. braziliensis y L. mexicana sp.

Fueron utilizados como fuente de colecta del material experimental, 60 hamsteres de nuestro bioterio de animales inoculados con cepas de Leishmania. Todos los animales presentaban nódulos sub-cutaneos no ulcerados e con úlceras recientes, parasitológicamente confirmados por la utilización de xenodiagnósticos con hembras de Lutzomyia whitmani ${ }^{6}$.

En sucesivos experimentos (I-VI) tres procedimientos fueron empleados para conocer su eficiencia relativa correspondiente en la colecta del material de las lesiones, en los animales. Asi mismo, 4 medios de cultivo (gentamicina $50 \mu \mathrm{g} / \mathrm{ml}$ ) fueron comparados en su eficiencia y sensibilidad. Procedimientos: a) De la aspiración, con aguja y jeringa, en la manera descrita por Hendricks y Wright ${ }^{13}$, pero ligeramente modificado por la utilización de una aguja de calibre mayor ( $22 \mathrm{~g}=25 \times 8 \mathrm{~mm}$ ) y una jeringa de $5 \mathrm{ml}$ (más potente en succión). b) De la micropipeta Pasteur, de punta muy afilada, esteril, que era introducida en los bordes de las heridas o en los nódulos subcutáneos, de los animales parasitados. El material colectado con $0,2 \mathrm{ml}$ de suero fisiológico $(0.85 \%)$ estéril era, entonces, sembrado en forma aséptica en dos tubos de cada medio investigado. c) La biopsia con "punch" de $4 \mathrm{~mm}$ de diámetro, que posteriormente era triturada con la ayuda de un "tissue grinder". La suspensión de tejidos era entonces inoculada como relatado anteriormente en los cultivos.

Un experimento (E4) utilizó la 5-fluorocy tosine
$(150 \mu \mathrm{g} / \mathrm{ml})$ en un grupo de tubos de cultivo DAB a ser comparados con medios $\mathrm{DAB}$ sin antifúngico. Todos los cultivos eran entonces mantenidos a $23^{\circ} \mathrm{C}$ y exámenes consecutivos a las $48,72,96$ y 120 horas fueron realizados con microscopio invertido. Posteriores exámenes a los 15 y 30 días fueron enseguida ejecutados. Con ésto pensamos establecer el periodo mínimo y máximo en la positivación de los cultivos inoculados.

3. Estudio del crecimiento en masa de promastigotes de cepas de $L$. b. braziliensis

3.1. Empleo de médios de cultivo líquido semidefinidos:

Los medios empleados fueron: TC 199 y de Schneider (Drosophila medium), ambos suplementados con 15 y $20 \%$ de suero bovino fetal. Estos medios fueron escogidos por ser líquidos y transparentes 17 .

El procedimiento consistió en la utilización de frascos plásticos de $25 \mathrm{ml}$ (Corning, USA), conteniendo, cada uno de ellos $5 \mathrm{ml}$ del medio líquido a experimentarse. La muestra de Leishmania a estudiarse era ajustada en una concentración de $1 \times 10^{6}$ promastigotes por $\mathrm{ml}$, en un volumen de $100 \mathrm{ml}$ del medio y entonces inoculada asépticamente. El recuento del número aproximado de promastigotes $/ \mathrm{ml}$ fue hecho en experimentos por duplicado y con la ayuda de una cámara de Neubauer y microscopio de contraste de fases. La observación del crescimiento en los frascos de cultivo fué realizada, sin necesidad de abrir los recipientes, utilizando un microscopio estereoscopio invertido Zeiss-Jena. Todos los "stocks" aislados fueron rápidamente criopreservados, luego del primeiro o segundo pesaje "in vitro" $y$, después de codificados, depositados en nuestro criobanco de cepas de Leishmania, a $-196^{\circ} \mathrm{C}$.

Los detalles laboratoriales de las cepas utilizadas en éste estudio figuran en la Tabla 1.

Tabla 1 - Caracteristicas biológicas y bioquímicas de cepas de Leishmania braziliensis braziliensis utilizadas en los experimentos de cultivo in vitro.

\begin{tabular}{|c|c|c|c|c|}
\hline $\begin{array}{l}\text { Código de la cepa } \\
\text { (Criobanco-Brasilia) }\end{array}$ & $K-D N A(2,3)$ & $\begin{array}{l}\text { Analisis por: } \\
\text { Isoenzimas (7) }\end{array}$ & $\begin{array}{l}\text { Anticuerpos (7) } \\
\text { Monoclonales }\end{array}$ & $\begin{array}{l}\text { Patrón del ( } 7) \\
\text { Desarrollo en } \\
\text { Flebótomo }\end{array}$ \\
\hline MHOM/BR/76/LTB-0014(L)* & + & + & + & + (Perypilaria) \\
\hline MHOM/BR/77/LTB-0014(R) & + & + & + & + (Perypilaria $)$ \\
\hline MHOM/BR/77/LTB-0042 & + & + & + & + (Perypilaria $)$ \\
\hline MHOM/BR/78/LTB-0048 & + & + & + & + (Perypilaria $)$ \\
\hline MHOM/BR/79/LTB-107 & + & + & N.H. & + (Perypilaria) \\
\hline MHOM/BR/78/C-25 & + & + & + & + (Perypilaria) \\
\hline MHOM/BR/79/C-31 & + & + & N.H. & + (Perypilaria $)$ \\
\hline
\end{tabular}

N.H. = no hech,

* Cepa de referencia internacional WHO (24). 
Netto EM, Cuba CC, Marsden PD, Barreto AC. El método de cultivo "in vitro" como instrumento práctico en el diagnóstico yel aislamiento primário de Leishmania braziliensis braziliensis. I. Observaciones de laboratorio. Revista da Sociedade Brasileira de Medicina Tropical 19: 79-84, Abr-Jun, 1986

\section{Análisis Estadístico}

El test exacto de Fischer (modificado por Tocher) fué utilizado en el análisis de las diferencias entre las proporciones obtenidas en los tests comparativos, con un nivel de significancia de $p<0,05$.

\section{RESULTADOS}

\section{1) Aislamiento primario de Leishmania de hamsteres infectados.}

La Tabla 2 presenta resultados que evidencian significativa positividad a favor del medio DAB, cuando comparado con el de Schneider (E 1). En un segundo experimento de comparación del $\mathrm{DAB}$ con el medio de Evans, confirmamos la eficiencia del DAB, siendo que el difásico de Evans también mostró similar sensibilidad, en el aislamiento primario de Leishmania braziliensis braziliensis (E 2). Un único aislamiento fué conseguido con el medio semidefinido AR-103 que se mostró poco apropriado para el cultivo in vitro de $L$. b. braziliensis (E 3).

En relación a la precocidad de crescimiento de los promastigotes de esa subespecie de Leishmania, $63 \%$ de los cultivos fueron positivos a las 48 horas de inoculados. Este tiempo de positivación fué verificado consistentemente en las posteriores observaciones efectuadas en los medios difásicos (DAB y Evans). Igualmente, en el medio de Schneider, $69 \%$ de los cultivos fueron positivos a las $\mathbf{4 8}$ horas de inoculados. A los 4 días, todos los tubos de cultivo que finalmente resultaron positivos ya presentaban promastigotes visibles al microscopio invertido.

Una otra observación (E 4) mostró que la adición de 5-fluorocytosine $(150 \mu \mathrm{g} / \mathrm{ml}$ de medio) como antifúngico no inhibió la diferenciación y multiplicación in vitro de L. b. braziliensis y L. mexicana sp., aisladas de 10 hamsteres infectados.

Finalmente, en otro experimento cuyo procedimiento fué la biopsia y triturado del material, realizado paralelamente al Experimento III se verificó que el medio AR-103 fracaso totalmente, en el aislamiento de $L b b$ de los 14 hamsteres infectados (E 5). Sin embargo, la misma observación demonstraba que el medio DAB estableció $92 \%$ de aislamiento primario del parásito.

Los resultados obtenidos por la succión del material de las lesiones con pipeta Pasteur (E 6) demuestran que $84 \%$ de los cultivos en 19 hamsteres fueron positivos cuando utilizado el medio DAB, siendo que solamente $5,3 \%$ de los tubos de Schneider fueron positivos en los mismos animales.

2) Crescimiento, en masa, de cepas seleccionados de L. b. braziliensis.

Comprobamos la necesidad del empleo de diversos lotes de suero bovino fetal (Gibco, Flow, Hyclone) en su capacidad de permitir el crecimiento in vitro de $L b b$. El suero bovino fetal del laboratorio Steryle System Hyclone (USA), demostró los mejores resultados cuando complementó, los medios líquidos de cultivo de tejidos animales TC-199 y de células de insectos de Schneider. Promastigotes, en densidades de $10^{8} / \mathrm{ml}$, fueron consistentemente desarrollados en ambos medios. La Tabla 3 muestra que los promastigotes de cepas humanas y canina de $L b b$ crecieron y multiplicaron en densidades de $10^{8}$ flagelados por ml de medio entre los días $5 \circ$ y 8 \% de cultivo in vitro, cuando inoculados los frascos de medio de Schneider con $10^{6}$ promastigotes $/ \mathrm{ml}$. Similares resultados (no presentados) fueron documentados utilizando el medio TC-199 (15\% S.B.F.).

Tabla 2 - Resultados de la comparación entre medios y procedimientos de cultivo en el aislamiento primario de Leishmania de hamsteres experimentalmente infectados.

\begin{tabular}{|c|c|c|c|c|c|c|c|c|}
\hline \multirow{2}{*}{$\begin{array}{l}\text { Médios y Experimentos } \\
\text { Experimentos }\end{array}$} & \multirow[t]{2}{*}{ Procedimientos } & \multicolumn{4}{|c|}{ Resultados* } & Total $(N)$ & \multicolumn{2}{|c|}{ Positividad } \\
\hline & & \multicolumn{5}{|c|}{$(++)(+-)(-+)(--)$} & & $(\text { valor } \mathrm{p})^{* *}$ \\
\hline E1) DAB x Schneider's & Aspir & 13 & 4 & 0 & 2 & 19 & $89,5 x$ & $68,4(\mathrm{p}<0,05)$ \\
\hline E2) DAB $\times$ Evans & con & 19 & 0 & 2 & 1 & 22 & $86,4 x$ & $95.5(\mathrm{p}>0,05)$ \\
\hline E3) DAB x AR-103 & Aguja y & 1 & 10 & 0 & 3 & 14 & $77,6 x$ & 7,1 \\
\hline E4) DAB $\times$ DAB & Jeringa & 10 & 0 & 0 & 0 & $10 * * *$ & $100,0 \times$ & 100,0 \\
\hline E5) DAB x AR-103 & Triturado & & & & & & & \\
\hline \multirow{3}{*}{ E6) DAB x Schneider's } & Biopsia & 0 & 13 & 0 & 1 & 14 & $92,0 \mathrm{x}$ & 0,0 \\
\hline & Aspiración con & & & & & & & \\
\hline & Pipeta Pasteur & 1 & 15 & 0 & 3 & 19 & $89,0 \mathrm{x}$ & 5.3 \\
\hline
\end{tabular}

* Los simbolos entre paréntesis indican $=(++)$, positividad por ambos medios; $(+-)$, positividad por el primer medio, DAB; $(-+)$, positividad por el segundo medio comparado; $(--)$, negatividad por ambos medios comparados.

** Diferencia estadistica significativa $\mathrm{p} \leq 0,05$. Test exacto de Fischer (Modificado por Tocher).

*** Solamente 5 de estos 10 animales estaban infectados con $L$. mexicana sp., el resto con L. b. braziliensis. 
Netto EM, Cuba CC, Marsden PD, Barreto AC. El método de cultivo "in vitro" como instrumento práctico en el diagnóstico y el aislamiento primário de Leishmania braziliensis braziliensis. I. Observaciones de laboratório. Revista da Sociedade Brasileira de Medicina Tropical 19: 79-84, Abr-Jun, 1986

Table 3 - Número de promastigotes de Leishmania braziliensis braziliensis cultivados en el medio de Schneider $(20 \%$ de suero bovino fetal $)-23^{\circ} \mathrm{C}$.

\begin{tabular}{lrrrc}
\hline & \multicolumn{4}{c}{ Número Aproximado de Flagelados/100 $\mu$ lde Medio } \\
\cline { 2 - 5 } Código de la Cepa & \multicolumn{1}{c}{ Inóculo } & 5 to día & 6to día & 8to día \\
\hline MHOM/BR/76/LTB-0014(L) & $2,17 \times 10^{6}$ & $1,52 \times 10^{8}$ & $1,51 \times 10^{8}$ & - \\
MHOM/BR/77/LTB-0014(R) & $2,7 \times 10^{6}$ & $0,12 \times 10^{8}$ & $0,4 \times 10^{8}$ & $2,18 \times 10^{8}$ \\
MHOM/BR/77/LTB-0042 & $5,1 \times 10^{6}$ & $1,77 \times 10^{8}$ & $1,89 \times 10^{8}$ & N.H. \\
MHOM/BR/78/LTB-0048 & $3,15 \times 10^{6}$ & $2,57 \times 10^{8}$ & $1,29 \times 10^{8}$ & N.H. \\
MHOM/BR/78/C-25 & $2,7 \times 10^{6}$ & $0,21 \times 10^{8}$ & $0,23 \times 10^{8}$ & $2,33 \times 10^{8}$ \\
\hline
\end{tabular}

N.H. $=$ no hecho

$-=$ contaminado

\section{DISCUSION}

En el transcurso de los últimos 3 años ha sido nuestro principal interés en la investigación de leishmaniasis, el cultivo in vitro de $L b b$ como técnica aplicada al diagnóstico precoz y, consecuentemente, al rápido aislamiento primário del parásito de pacientes en las áreas endémicas de Bahia, Brasil ${ }^{8}$. El éxito en el cultivo en masa de los promastigotes de ésta subespecie de Leishmania, por otro lado, benefició directamente los estudios de identificación taxonómica.

Un paso preliminar al amplio empleo de los medios artificiales de cultivo, en estudios de campo, ha sido evaluar la aplicación de los medios difásicos y líquidos, aparentemente promisorios, publicados en la literatura 41214 en tentativas de aislamiento de cepas, bien caracterizadas de $L b b$ y $L$. mexicana, mantenidas en nuestro laboratorio. Este procedimiento laboratorial ya fue empleado por Werner ${ }^{23}$, y Chavez et alii $^{5}$ y constituye ayuda valiosa sobretodo en el estudios de las infecciones causadas por $L b b$.

Analizando la metodologia descrita en otras investigaciones comprobamos que, el procedimento de colecta del material infectado consistió en la aspiración de los amastigotes, empleando una aguja $27 \mathrm{~g} 13$ $o$, por la utilización de una jeringa de tuberculina munida a una aguja de n. $23 \mathrm{~g}^{5}$. Nuestro procedimiento utilizando una jeringa de $5 \mathrm{ml}$ y aguja no $22 \mathrm{~g}$, demostró buena eficiencia tanto en la fuerza de succión como en la obtención de razonable volumen de tejidos retirados de las lesiones. A éste último detalle se adiciona la buena sensibilidad verificada con el medio agar sangre Difco, tal como lo recomendado por Walton $e t$ alii 22 y Shaw \& Lainson 20.

Avances relativamente recientes han sido efectuados en el cultivo de los promastigotes de Leishmania, por el uso de medios de cultivo de células de mamíferos e insectos, complementados com suero bovino fetal 17 . Algunos investigadores afirman que los medios líquidos son superiores a los difásicos (básicamente de agar sangre) en el aislamiento primario de Leishmania, tanto de pacientes como de animales experimentales 1314 . A pesar del medio de Evans haber demostrado eficiencia similar al DAB, el empleo de lotes diferentes de sangre de caballo pareció influenciar en el rendimiento del primero. Por esa razón resultó más práctico el empleo del $\mathrm{DAB}$ que utiliza sangre de conejo desfibrinada de más fácil obtención en nuestro medio. Esto confirma los resultados obtenidos por los investigadores de Pará, Brasil ${ }^{20}$, Bolivia 21 y Perú ${ }^{15}$, con cepas de $L$. braziliensis.

Una rapida conversión in vitro para promastigotes fué observada en un periodo tan corto como 48 horas de inoculado el medio DAB. Este desempeño fué mejor que el obtenido por Chavez et alii ${ }^{5} \mathrm{y}$ al emplear el medio Senekjie. Así, mismo, en el medio $\mathrm{DAB}, L b b$ vive tanto como 45 dias y, rutinariamente, es posible subcultivar el parásito cada 25 días. Esto último contrasta con el comportamiento del parásito en el medio de Schneider en el cual al pico de crecimiento es alcanzado rápidamente en la primeira semana de cultivo "in vitro", después del cual los parásitos mueren entre el $12 \%$ y $15 \%$ día.

Por otro lado medios que son eficientes en el aislamiento de Leishmania puedem no ser igualmente apropriados para el cultivo en gran escala de los organismos y vice-versa ${ }^{10}$. Las cepas padrón de $L b b$ estudiadas demonstraron consistente crecimiento y multiplicación "in vitro" en los medios líquidos de TC-199 y de Schneider. Fué posible, de esta manera cultivar esos parasitos en cantidad suficiente de células para la realización de estudios de taxonomia bioquímica ${ }^{3}$. Estos hallazgos amplian las observaciones verificadas con otras especies de Leishmania ${ }^{14}$, confirmando las ventajas obvias del uso de medios líquidos transparentes. 
Netto EM, Cuba CC, Marsden PD, Barreto AC. El método de cultivo "in vitro" como instrumento práctico en el diagnóstico yel aislamiento primário de Leishmania braziliensis braziliensis. I. Observaciones de laboratório. Revista da Sociedade Brasileira de Medicina Tropical 19: 79-84, Abr-Jun, 1986

\section{SUMMARY}

We have compared different media and isolation procedures to cultivate Leishmania braziliensis braziliensis from hamster tissues. We find that aspiration of the lesion using a $22 \mathrm{~g}$ needle and a five $\mathrm{ml}$ syringe gives satisfactory results, and is a convenient sampling method. Difco Blood agar is an efficient medium for isolation. Evans medium gave equal results while Schneider's and AR-103 medium were inferior. In Schneider's medium flagellate growth achieved $0.21-2.57 \times 10^{8}$ promastigotes $/ \mathrm{ml}$ by the fifth day. 5-fluorocytosine added to the cultures to avoid fungal contamination does not inhibit the growth of $\mathrm{L}$. b. brasiliensis.

Key' words: Leishmania braziliensis braziliensis. Culture in vitro.

\section{REFERÊNCIAS BIBLIOGRÁFICAS}

1. Azevedo JP, Roitman, I. Cultivation of Trypanosoma cruzi in defined media. In: Genes and Antigens of parasites. A laboratory manual. Morel C. (ed.) 2nd Edition UNDP/World Bank/WHO Special Programme for Research and Training in Tropical Disease. Rio de Janeiro, pp. 29-33, 1984.

2. Barker DC. Butcher. J. The use of DNA probes in the identification of leishmaniasis discrimination between isolates of the Leishmania mexicana and L. braziliensis complexes. Transactions of the Royal Society of Tropical Medicine and Hygiene 77:285-297, 1983.

3. Barker DC, Butcher J, Gibson LJ, Kennedy WPK, Williams RH, Cuba Cuba CA, Marsden PD, Lainson R, Shaw JJ. Sequence homology of kinetoplast DNA in Leishmania studied by filter hybridisation of endonuclease digested fragments and "in situ" hybridisation of individual organisms Annales de Parasitologie. in press

4. Chang KP, Fish WR Leishmania. In: In vitro cultivation of protozoa pathogenic to man and domestic animals. Jensen J.B. (ed.) CRE, Press, Florida. pp. 2-91, 1981.

5. Chaves F. Silva S, Zeledon R. Comparison of two culture media for the isolation of Leishmania strains. The Journal of Parasitology 68:346-347, 1982.

6. Cuba Cuba CA, Vexenat AJ, Llanos-Cuentas EA, Marsden PD, Barreto AC, Rosa AC. Experimental infections of wild caught specimens of Lutzomyia $(N)$ whitmani (Diptera, Psychodidae) and their use for Leishmania identification. In: Resumos da IX Reuniāo Anual Pesquisa Básica em Doença de Chagas. Caxambú. MG, Brasil, 1982.

7. Cuba Cuba, CA. Leishmaniose Tegumentar em área endèmica do Estado da Bahia, Brasil. Caracterização e classificação de Leishmania do homem e do cào doméstico e aspectos comportamentais de L. braziliensis braziliensis. Tese de Doutorado. Universidade Federal de Minas Gerais. Brasil. 152 pp.. 1983.

8. Cuba Cuba CA, Netto EM, Costa JML, Rosa AC, Barreto AC, Marsden PD. Cutaneous leishmaniasis caused by Leishmania braziliensis braziliensis. Improving diagnosis by primary isolation in culture. Resumo No I-70. XI Reunião Anual Pesquisa Básica em Doença de Chagas, Caxambú, MG. Brasil, 1984.

9. Cuba Cuba CA, Llanos-Cuentas EA, Barreto AC, Magalhães AV, Lago EL, Reed SG, Marsden PD. Human mucocutaneous leishmaniasis in Très Braços, Bahia, Brazil. An area of Leishmania braziliensis braziliensis transmission. I. Laboratory Diagnosis. Revista da Sociedade Brasileira de Medicina Tropical 17:161-167, 1984.

10. Evans, D. The isolation and cultivation of Leishmania in vitro. In Barker J.. Taylor. A. (ed.). 2nd edition. Academic Press. pp. 82-83. 1979

11. Evans DA, Lanham SM, Baldwin C, Peters W. The isolation and isoenzyme characterization of Leishmania braziliensis sub sp. from patients with cutaneous leishmaniasis acquired in Belize. Transactions of the Royal Society of Tropical Medicine and Hygiene 78:35-42. 1984.

12. Hendricks LD, Wood DE, Hadjuk ME. Haemoflagellates: commercially available liquid media for rapid cultivation. Parasitology 76:309-316. 1978.

13. Hendricks LD, Wright N. Diagnosis of cutaneous leishmaniasis by in vitro cultivation of saline aspirates in Schneider's Drosophila medium. American Journal of Tropical Medicine and Hygiene 28:962-964, 1979.

14. Hendricks LD, Childs GE. Present knowledge of the in vitro cultivation of Leishmania. In: In vitro cultivation of major pathogens of tropical disease. Tropical Disease Research Series 3:251-271. Basel. Schwabe \& Co, 1980.

15. Llanos-Cuentas A. Arana M, Montoya Y, Campos M, Romero G. Aislamiento primario de Leishmania sp. de pacientes en area endemicas del Perú. Resumo BI-67. XI Reunião Anual Pesquisa Básica em Doença de Chagas, Caxambú, MG, Brasil, 1984.

16. Mayrink W, Raso P, Melo MN, Michalick MSM, Magalhães PA. Williams P. Costa CA. Dias M. American cutaneous leishmaniasis. Disappearance of amastigotes from lesions during antimonial therapy. Revista do Instituto de Medicina Tropical de Sāo Paulo 25:265-269, 1983.

17. Miles MA. Leishmania culture and biochemical comparisons. Some difficulties. In: Biochemical Characterization of Leishmania. Proceedings Workshop PAHO, Washington D. C. Chance M. \& Walton B. C. (ed.) UNDP/World/Bank/WHO, pp. 124-137, 1982.

18. Palomino JC. Guerra H. Lumbreras H. A selective liquid medium for primary isolation of South American Leishmaniasis. Tropenmedizin und Parasitologie 34:229-232, 1983

19. Peters W, Evans DA Lanham SM. Importance of parasite identification in cases of leishmaniasis. Journal of the Royal Society of Medicine 76:540-542. 1983.

20. Shaw JJ, Lainson $R$. The in vitro cultivation of members of the Leishmania braziliensis complex. Transactions of the Royal Society of Tropical Medicine and Hygiene $75: 127,1981$ 
Netto EM, Cuba CC, Marsden PD, Barreto AC. El método de cultivo "in vitro" como instrumento práctico en el diagnóstico yel aislamiento primário de Leishmania braziliensis braziliensis. I. Observaciones de laboratório. Revista da Sociedade Brasileira de Medicina Tropical 19: 79-84, Abr-Jun, 1986

21. Urjel R. Recacoechea M, La Fuente C, Orellana H. A simple method for the collection of material from cutaneous and mucocutaneous leishmaniasis lesions. Transactions of the Royal Society of Tropical Medicine and Hygiene 77:882-883, 1983.

22. Walton BC, Shaw JJ, Lainson R. Observations on the in vitro cultivation of Leishmania braziliensis. Journal of Parasitology 63:1118-1119, 1977.
23. Werner JK. Colombian strains of Leishmania from man: growth characteristics in culture media and hamsters. Transactions of the Royal Society of Tropical Medicine and Hygiene 75:619-622, 1981.

24. World Health Organization - International reference strains of Leishmania. Bulletin of the World Health Organization 63:43-46, 1985. 